\title{
Emergence of Selectivity in Inherently Nonselective Gold Nanoparticles through Preferential Breaking of Interparticle Interactions
}

\author{
Anish Rao, Govind S., Soumendu Roy, Ajesh T. R., Gayathri Devatha and Pramod P. Pillai* \\ Department of Chemistry and Center for Energy Sciences, Indian Institute of Science Education and \\ Research (IISER), Dr. Homi Bhabha Road, Pashan, Pune - 411008, India \\ *Correspondence to: pramod.pillai@iiserpune.ac.in
}

\section{$\underline{\text { TOC Graphic }}$}

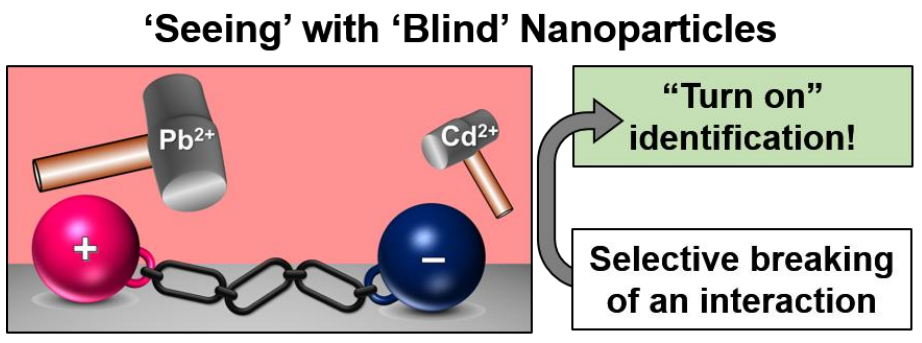

\begin{abstract}
Establishing a 'precise' control over different interparticle interactions holds the promise of introducing inherently absent properties to nanosystems. In this direction, we demonstrate a conceptually unique strategy to introduce the notion of selectivity in inherently nonselective carboxylate-functionalized gold-nanoparticles ([-] AuNP), towards strongly binding divalent metal ions $\left(\mathrm{M}^{2+}\right)$. The present system designed from such nonselective nanoparticles is able to discriminate between $\mathrm{M}^{2+}$ ions, by using differences in their abilities to break interparticle interactions. This is in stark contrast with the conventional identification protocol of forming an interaction between NPs and $\mathrm{M}^{2+}$ ions, with the help of analyte-specific ligands. Among different ions tested $\mathrm{Pb}^{2+}$ ion was preferentialy able to break the electrostatic interactions in $[+]-[-]$ Au nanoionic precipitates and displace [+] AuNP to solution, thereby turning on the plasmonic wine-red color. The dominance of interaction energy for [-] AuNP - $\mathrm{Pb}^{2+}$ complexation over the inter-nanoparticle interactions is accountable for the selective discrimination of $\mathrm{Pb}^{2+}$ from other $\mathrm{M}^{2+}$ ions. A careful, yet convenient, variation in the strength of different interparticle interactions helped in tuning both the selectivity and sensitivity of our identification protocol.
\end{abstract}




\section{Introduction}

Development of strategies to improve and impart newer properties to existing materials, without the need of new components, is one of the future directions in modern nanoscience. ${ }^{1}$ In this regard, strategies based on controlling the forces at nanoscale ${ }^{2-4}$ are promising due to its already proven impact in self-assembly, ${ }^{5-10}$ catalysis, ${ }^{11,12}$ light harvesting, ${ }^{13}$ and biotargeting. ${ }^{14-21}$ The present work uses judiciously designed interparticle interactions to carve out new properties from pre-existing materials. We demonstrate the decisive role of forces in introducing the notion of selectivity in inherently nonselective carboxylate-functionalized gold-nanoparticles ([-] AuNP), towards strongly binding divalent metal ions $\left(\mathrm{M}^{2+}\right)$. Overcoming nonselectivity is one of the long existing challenges in the area of [-] AuNP sensors: ${ }^{22-25}$ a case analogous to the solvent levelling effect ${ }^{26}$ where the solvent fails to discriminate between different strong acids or bases. The levelling in [-] AuNP arises because of the strong abilities of $\mathrm{M}^{2+}$ ions to bridge the carboxylate groups (Fig. 1a). ${ }^{22-25}$ Here the strong bridging interaction triggers the nonselective aggregation and plasmon coupling in NPs causing a rapid colour change, ultimately leading to precipitation (turn-off response). ${ }^{22-25}$ Essentially, a dispersed solution of NP is an example of a kinetically trapped or thermodynamically less stable state because of the large number of high energy surface atoms with unsatisfied valences. ${ }^{27,28}$ The number of contacts between the nanoparticles increases during the process of aggregation/precipitation, which leads to a decrease in the number of high energy surface atoms with unsatisfied valences. ${ }^{28}$ Thus, the aggregated state of NP is thermodynamicaly more stable when compared to the dispersed state. Introduction of external stimuli (like $\mathrm{M}^{2+}$ ions) can thus uniformly trigger the thermodynamically favourable process of precipitation, imparting the nonselectivity to [-] AuNPs. The common practice to overcome such nonselectivity is to replace the carboxylate groups on [-] AuNPs with analytespecific ligands. However, most of the reported analyte-specific nanohybrid systems exhibit turn-off response by ultimately precipitating out, ${ }^{29-37}$ with the exception of a few turn-on systems. $^{38,42}$

We report a conceptually unique strategy to achieve the selective turn-on identification of heavy metal ions, with AuNPs that are deprived of any analyte-specific ligands. Our approach is to explore the differences in the abilities of $\mathrm{M}^{2+}$ ions to interact with a thermodynamically stable ${ }^{27,28}$ inter-nanoparticle precipitate containing [+] and [-] AuNPs (Fig. 1b). Here we emphasize that both [+] and [-] AuNPs, independently, were nonselective towards $\mathrm{M}^{2+}$ ions. Remarkably, a system composed of such nonselective nanoparticles was able to discriminate between the hard-to-distinguish pair of $\mathrm{Pb}^{2+}$ and $\mathrm{Cd}^{2+}$ ions. The rationale 
is that only the strongest of strongly binding ions will be able to break the interactions in nanoionic precipitates (thermodynamically stable state), ${ }^{27,28}$ and disperse them back to the solution (kinetically trapped state) ${ }^{27}$.

\section{a) Formation of an interaction: Nonselective "turn-off" response}

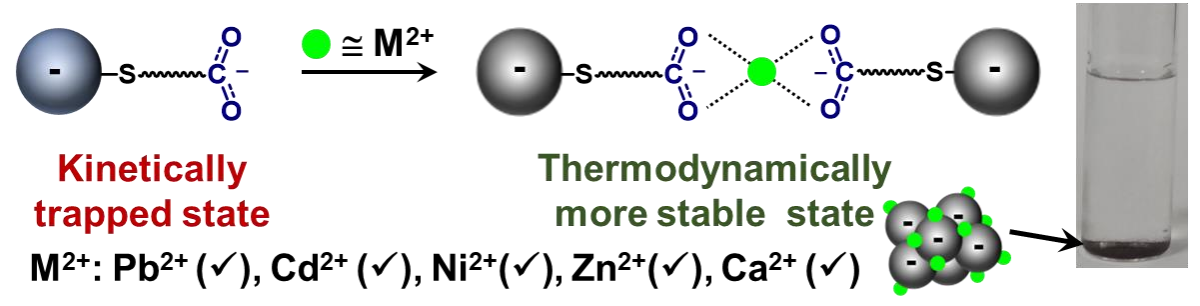

b) Breaking of an interaction: Selective "turn-on" response

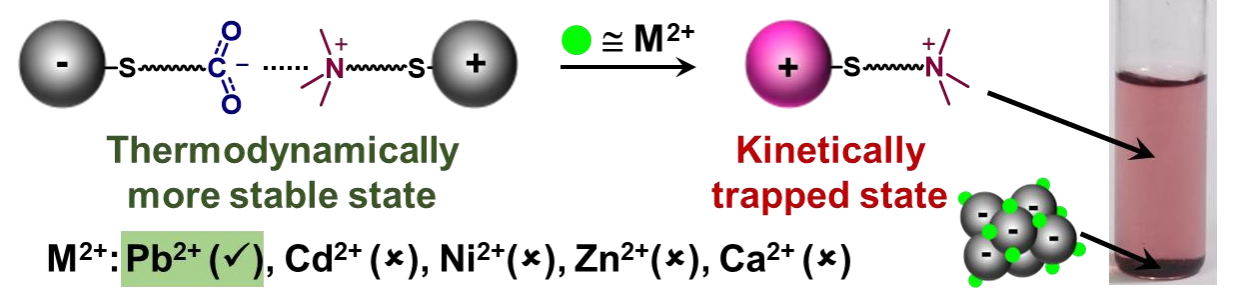

Figure. 1 Schematics for selective turn-on response with [-] AuNPs. (a) The kinetically trapped [-] AuNPs coordinate with various $\mathrm{M}^{2+}$ ions uniformly to reach the thermodynamically more stable precipitate state, resulting in the inherent nonselective turnoff response. (b) Our hypothesis to explore differences in the abilities of $\mathrm{M}^{2+}$ ions to break an interaction as the means of discrimination, rather than the conventional idea of forming of an interaction.

We worked with the nanoionic precipitates having oppositely charged [+] and [-] AuNPs stitched together through electrostatic and van der Waals attractions, developed by Grzybowski and coworkers. ${ }^{43}$ The concept of preferential breaking of interactions in $[+]-[-]$ $\mathrm{Au}$ nanoionic precipitates by $\mathrm{M}^{2+}$ ions, led to the displacement and leaking of [+] AuNPs, providing the desired turn-on selectivity (Fig. 1b). Only $\mathrm{Pb}^{2+}$ ion was capable of breaking the electrostatic interactions in [+] - [-] Au nanoionic precipitates, among other $\mathrm{M}^{2+}$ ions including the $\mathrm{Cd}^{2+}$ ions. The sensitivity and selectivity of nanoionic precipitates were tuned by controlling the strength of electrostatic interactions between the NP constituents. The sensitivity of $\mathrm{Pb}^{2+}$ ions was improved from $1 \mathrm{mM}$ to $20 \mu \mathrm{M}$ by lowering the electrostatic attractions in the nanoionic precipitate formed from heterogeneously charged $[+/-]_{9}$ and homogenously charged [+] AuNPs. More importantly, $3 \mathrm{mM}$ of $\mathrm{Cd}^{2+}$ ion was also able to break the electrostatic interactions between $[+]-[+/-]_{9}$ Au nanoionic precipitates, proving the tunability in $\mathrm{M}^{2+}$ ion detection as per demand. 


\section{Results and Discussion}

\section{Selective turn-on response of [+] - [-] Au nanoionic precipitates}

The AuNP systems with a core diameter of $5.5 \pm 0.7 \mathrm{~nm}$ and varying surface chemistries were prepared by adopting a modified literature report. ${ }^{23,44,45}$ Non-ionizable N,N,Ntrimethylmercaptoundecyl ammonium chloride (TMA, [+]) and ionizable 11mercaptoundecanoic acid (MUA, [-]) ligands were functionalized on AuNPs to impart positive and negative surface charges, respectively (Figs. S1 and S2, ESI $\dagger$ ). The aggregation process of different AuNP systems in the presence of various $\mathrm{M}^{2+}$ ions was monitored using time-dependent UV-Vis absorption studies. As reported previously, [-] AuNPs complexed and ultimately precipitated with different $\mathrm{M}^{2+}$ ions, confirming their inherent nonselectivity (Fig. S3, ESI $\dagger$ ). ${ }^{22-25}$ To overcome this, our approach was to use the abilities of different $\mathrm{M}^{2+}$ ions to break the interactions in inter-nanoparticle precipitates (thermodynamically more stable state), ${ }^{27,28}$ and disperse them back to the solution (kinetically trapped state) ${ }^{27}$. Accordingly, the inter-nanoparticle precipitates containing [-] AuNPs were prepared from an equimolar mixture of [+] and [-] AuNPs (Fig. 2a), as reported by Grzybowski and coworkers. ${ }^{43}$ The addition of small ( 0.15 equivalents) aliquots of [-] AuNPs to [+] AuNPs triggers the aggregation and plasmon coupling processes, through the strong electrostatic attraction. This is accompanied by a gradual bathochromic shift in the $\lambda_{\max }$ from $515 \mathrm{~nm}$ to $555 \mathrm{~nm}$, followed by an abrupt loss of plasmon band (Figs. S4 and S5, ESI $\dagger$ ). This is due to an ionic-like sharp precipitation of [+] and [-] AuNPs at the charge neutrality (i.e. when $\Sigma_{\mathrm{Q}[+]}$ $\left.+\Sigma_{\mathrm{Q}[-]}=0\right)$, confirming the formation of [+] - [-] Au nanoionic precipitates. ${ }^{43}$

Upon succeeding in the preparation of $[+]-[-]$ Au nanoionic precipitates, the breaking abilities of various strongly binding $\mathrm{M}^{2+}$ ions were systematically studied (Figs. 2 and S7, ESI $\dagger$ ). It was observed that the addition of $\mathrm{Pb}^{2+}$ ions to [+] - [-] Au nanoionic precipitates resulted in the revival of plasmon color to the solution. For instance, a clear reappearance of wine-red color was observed after the addition of $\sim 1 \mathrm{mM}$ of $\mathrm{Pb}^{2+}$ ions (limit of detection, LOD, green spectrum in Fig. 2b); while $\sim 85 \%$ of revival in the plasmon intensity was observed upon the addition of $\sim 3 \mathrm{mM}$ of $\mathrm{Pb}^{2+}$ ions (red spectrum in Fig. 2b). The redispersed solution contained black precipitates corresponding to [-] AuNP $-\mathrm{Pb}^{2+}$ aggregates as well. The photographs of the solution at each stage are presented in Fig. 2d, which clearly shows the revival of plasmon color and the sedimented precipitates. The Transmission Electron Microscope (TEM) images shown in Figs. 2e and S6 (ESI $\dagger$ ) prove the leakage of [+] AuNPs from nanoionic precipitates, where both individual [+] AuNP and [-] AuNP $-\mathrm{Pb}^{2+}$ 
precipitates are clearly visible. Interestingly, all the other $\mathrm{M}^{2+}$ ions $\left(\mathrm{Cd}^{2+}, \mathrm{Ni}^{2+}, \mathrm{Zn}^{2+}\right.$ and $\mathrm{Ca}^{2+}$ ), despite being capable of coordinating with individual [-] AuNPs, failed to revive the plasmon color under similar conditions, proving the selectivity of $[+]-[-] \mathrm{Au}$ nanoionic precipitates towards $\mathrm{Pb}^{2+}$ ions (Figs. 2c, 3a, S6, and S8-S11, ESI $\dagger$ ). Even a mixture of other $\mathrm{M}^{2+}$ ions $\left(\mathrm{Cd}^{2+}, \mathrm{Ni}^{2+}, \mathrm{Zn}^{2+}\right.$ and $\left.\mathrm{Ca}^{2+}\right)$ was unable to break the interparticle interactions in [+] [-] Au nanoionic precipitates (Figs. 3a, S8, ESI†). However, a revival of plasmon band was observed when $3 \mathrm{mM}$ of $\mathrm{Pb}^{2+}$ ions were included in the mixture (Figs. 3a, S8, ESI $\dagger$ ). The other important ions like $\mathrm{Hg}^{2+}$ and $\mathrm{Sn}^{2+}$ were not included in the present study due to their

a)

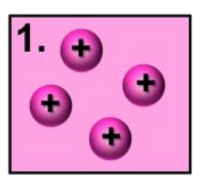

[+] AuNP b)

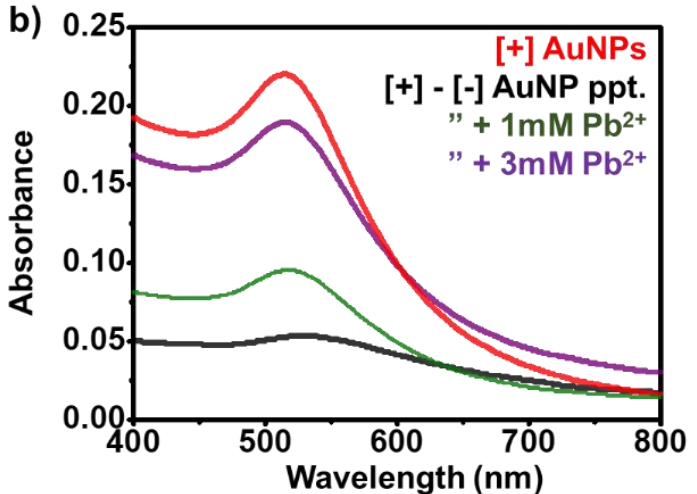

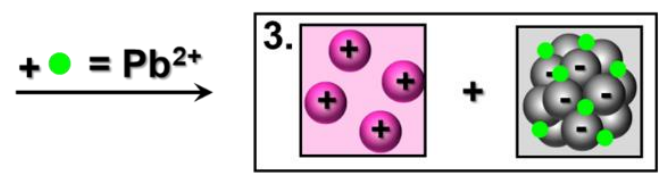

$[+]$ AuNP + [-] AuNP - $\mathrm{Pb}^{2+}$ ppt.

d)

1.

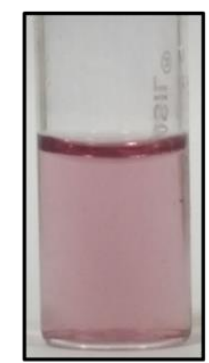

2.

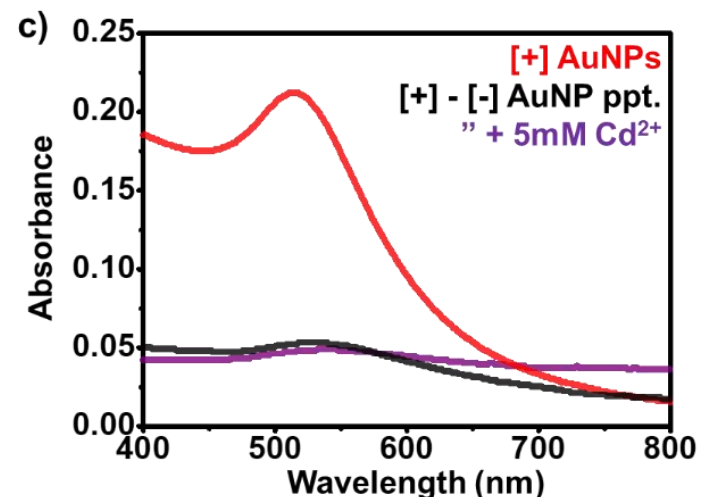

e)

3.
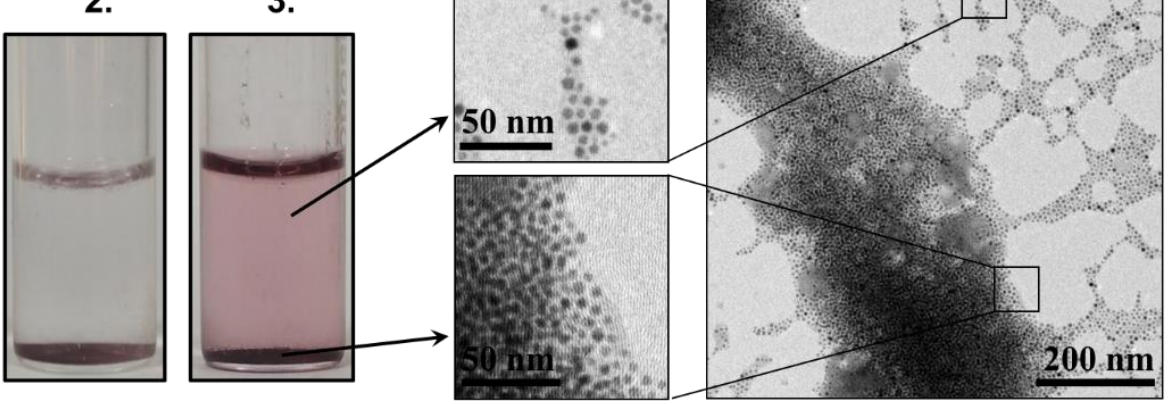

Figure. 2 Selective turn-on response of [+] - [-] Au nanoionic precipitates. (a) Schematics for the preparation of [+] - [-] Au nanoionic precipitates and the selective turn-on detection of $\mathrm{Pb}^{2+}$ ion. Variation in the absorption of $[+]-[-]$ Au nanoionic precipitates in the presence of (b) $\mathrm{Pb}^{2+}$ and (c) $\mathrm{Cd}^{2+}$ ions. An $\sim 85 \%$ revival of plasmon band was observed upon the addition of $3 \mathrm{mM}$ of $\mathrm{Pb}^{2+}$ (with LOD $=1 \mathrm{mM}$, green spectrum); whereas even $3 \mathrm{mM}$ of $\mathrm{Cd}^{2+}$ ions failed to break the [+] - [-] Au nanoionic precipitates. (d) Photographs of the vials corresponding to stages 1, 2 and 3 in Scheme (a). (e) A representative TEM image of [+] - [-] $\mathrm{Au}$ nanoionic precipitate after the addition of $3 \mathrm{mM}$ of $\mathrm{Pb}^{2+}$ ions. The enlarged TEM images on the left show the selected portions corresponding to dispersed [+] AuNPs and [-] AuNP $\mathrm{Pb}^{2+}$ precipitate. 
inappropriateness with the [+] - [-] Au nanoionic systems (See Section 6 in the ESI†). Further, a high concentration of $\mathrm{Cd}^{2+}(\sim 200 \mathrm{mM})$ was able to break the electrostatic interactions in $[+]-[-]$ Au nanoionic precipitates (Fig. S9, ESI $\dagger$ ). In comparison with $\mathrm{Pb}^{2+}$ ion, $\sim 200$ times excess of $\mathrm{Cd}^{2+}$ ion was required to revive $\sim 50 \%$ of plasmon color to the solution. This confirms that the nature as well as the strengths of interaction between [-] AuNP and $\mathrm{M}^{2+}$ ions forms a key step in our identification protocol.

\section{Validation of preferential breaking of interparticle interactions}

According to our hypothesis, the selectivity towards $\mathrm{Pb}^{2+}$ ions originates from the relative differences in the interaction strengths between [-] AuNPs and $\mathrm{M}^{2+}$ ion. To ascertain this, we have estimated the binding ability of various $\mathrm{M}^{2+}$ ions with [-] AuNP through two independent studies. Firstly, titration experiments were performed between [-] AuNP and aliquots of different aggregation triggers ([+] AuNP, $\mathrm{Pb}^{2+}, \mathrm{Cd}^{2+}$ and $\mathrm{Ca}^{2+}$; Fig. S12, ESI $\dagger$ ). Fig. 3b shows the variation in $\lambda_{\max }$ of [-] AuNP as a function of concentration of various aggregation triggers. The transition point $(\tau)$ that is estimated from the mid-point of the transition window signifies the minimum amount of titrant required to precipitate [-] AuNPs. The $\tau$ value describes the affinity between two interacting species, which is inversely proportional to (at least qualitatively) the magnitude of interaction energy. ${ }^{46}$ The $\tau$ values for different aggregation triggers were estimated to be in the following order: $\tau\left(\mathrm{Pb}^{2+}\right) \sim 50 \mu \mathrm{M}<$ $\tau([+]$ AuNP $) \sim 80 \mu \mathrm{M}<\tau\left(\mathrm{Cd}^{2+}\right) \sim 110 \mu \mathrm{M}<\tau\left(\mathrm{Ca}^{2+}\right) \sim 215 \mu \mathrm{M}$. The interaction energies between [-] AuNPs and $\mathrm{M}^{2+}$ ions, therefore, follows the reverse order: $\mathrm{Pb}^{2+}>[+] \mathrm{AuNP}>\mathrm{Cd}^{2+}>\mathrm{Ca}^{2+}$. The superior binding interactions between [-] AuNP and $\mathrm{Pb}^{2+}$ ions favors the displacement of [+] AuNP from nanoionic precipitates by $\mathrm{Pb}^{2+}$ ions, confirming our hypothesis. On the contrary, $\mathrm{Cd}^{2+}$ and $\mathrm{Ca}^{2+}$ ions failed to displace [+] AuNP from the nanoionic precipitates due to the higher $\tau$ values (and lower interaction energies). 

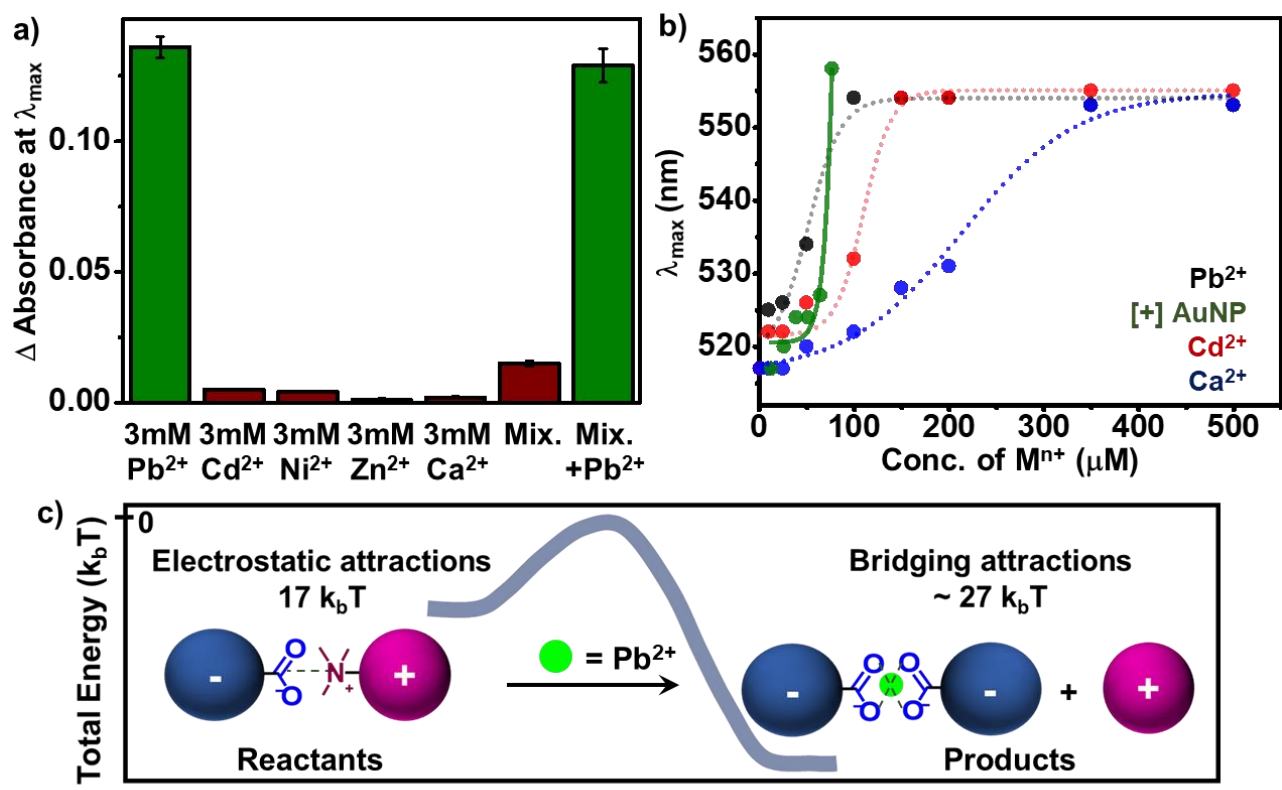

Reaction co-ordinate

Figure. 3 Selectivity of [+] - [-] Au nanoionic precipitates in identifying $\mathbf{P b}^{2+}$ ions. (a) Effect of different $\mathrm{M}^{2+}$ ions and mixture of ions on the absorbance of [+] - [-] Au nanoionic precipitates. A revival of the plasmon band $(\sim 85 \%)$ was observed only in the presence of $3 \mathrm{mM} \mathrm{Pb}{ }^{2+}$ ions, confirming the selective turn-on response. (b) Variation in the $\lambda_{\max }$ of [-] AuNP as a function of concentration of different aggregating triggers. The individual absorption spectra are shown in Fig. S12, ESI $\dagger$. The markers correspond to the experimental data, while the solid/dotted lines show the sigmoidal fits for the data. (c) Schematics showing the variation in energies of nanohybrid systems before and after the exposure to $\mathrm{Pb}^{2+}$ ions.

Secondly, different interaction energies between NPs and $\mathrm{M}^{2+}$ ions were estimated using theoretical modeling of different interparticle interactions. In this model, we compared total energies of the nanohybrid systems before and after the exposure to different $\mathrm{M}^{2+}$ ions (Fig. 3c) ${ }^{3,47,48}$ The key forces holding the $[+]$ - [-] Au nanoionic precipitates (reactants) are electrostatic and van der Waals interactions. Similarly, bridging and van der Waals interactions are the key forces responsible for [-] AuNP - $\mathrm{M}^{2+}$ complex formation (products). The detailed information about the process of modeling these interactions is given in Section 9 of the ESI†. Briefly, van der Waals attraction between two AuNPs in contact was modeled using Hamaker integral approximation, ${ }^{3}$ and was estimated to be $\sim 1.5 \times 10^{-21} \mathrm{~J}$ or $\sim 0.36 \mathrm{k}_{\mathrm{b}} \mathrm{T}$. The electrostatic interactions between charged AuNPs in ionic solution $\left(\mathrm{c}_{\mathrm{s}} \sim 1 \mathrm{mM}\right){ }^{49}$ were estimated by solving the electrostatic potential $(\varphi)$ via thermodynamic integration. ${ }^{49} \mathrm{We}$ then solved the Poisson-Boltzmann equation (while accounting for "charge-regulation") for two interacting spheres, ${ }^{3,50}$ and the electrostatic attraction energy between oppositely charged AuNP pair was estimated to be $\sim 6.2 \times 10^{-20} \mathrm{~J}$ or $\sim 17 \mathrm{k}_{\mathrm{b}} \mathrm{T}$. This is in close agreement with the values reported by Grzybowski and coworkers. ${ }^{3,49}$ The magnitude of the bridging interaction in [-] AuNP $-\mathrm{M}^{2+}$ complex was estimated by modifying a reported equilibrium model of the 
crosslinking interactions ${ }^{47}$ and using literature reported values for binding constants of $\mathrm{Pb}^{2+}$ and $\mathrm{Cd}^{2+}$ ions with acetate groups ${ }^{51,52}$. Based on the modeling studies, $\mathrm{Pb}^{2+}$ ions have the highest interaction energy with [-] AuNP $\left(\sim 1.1 \times 10^{-19} \mathrm{~J}\right.$ or $\left.\sim 27 \mathrm{k}_{\mathrm{b}} \mathrm{T}\right)$ followed by [+] AuNP $\left(\sim 6.2 \times 10^{-20} \mathrm{~J}\right.$ or $\left.\sim 17 \mathrm{k}_{\mathrm{b}} \mathrm{T}\right)$, and $\mathrm{Cd}^{2+}$ ions $\left(\sim 1.8 \times 10^{-20} \mathrm{~J}\right.$ or $\left.\sim 4.34 \mathrm{k}_{\mathrm{b}} \mathrm{T}\right)$. The estimated energy values re-iterate superior interaction of $\mathrm{Pb}^{2+}$ ions with [-] AuNP and their ability to displace $[+]$ AuNP from nanoionic precipitates, resulting in the desired selectivity.

\section{Versatility and tunability of identification}

Next we discuss the versatility and tunability in the identification ability of Au nanoionic precipitates. The selectivity stems from the preferential breaking of electrostatic interactions in [+] - [-] Au nanoionic precipitates. Thus, a variation in the strength of electrostatic forces can, in principle, tune the selectivity and sensitivity for different analytes of interest. Moreover, the presence of [-] AuNP $-\mathrm{Pb}^{2+}$ precipitate in the final solution is undesirable, and can be circumvented by decreasing the strength of bridging attractions in the complex. Accordingly, the nanoionic precipitates were prepared by using heterogeneously charged [+/] AuNP instead of homogenously charged [-] AuNP (Fig. 4a). The heterogeneously charged $[+/-]_{9}$ AuNP were synthesized by place exchange reaction with 1:9 mixture of $[+]$ and $[-]$ ligands. The on-NP ratio of [+]:[-] was estimated to be 1:7 using previously reported relative binding affinities of [+] and [-] ligands $\left(\mathrm{K}_{[+]} / \mathrm{K}_{[-]}=1.2\right)$, where $\mathrm{K}_{[+]}$and $\mathrm{K}_{[-]}$are the equilibrium constants for the adsorption of [+] and [-] onto AuNP respectively. ${ }^{23,53}$ Similar to the titration behavior of homogeneously charged AuNP, the mixture of $[+/-]_{9}$ and $[+]$ AuNPs precipitated sharply at the charge neutrality (Figs. 4b,c). Interestingly, $\sim 70 \%$ of $[+]$ AuNPs was sufficient to form the $[+]-[+/-]_{9}$ Au nanoionic precipitates compared to $[+]-[-] \mathrm{Au}$ nanoionic precipitates. This confirms the decrease in the strength of electrostatic attractions in $[+]-[+/-]_{9} \mathrm{Au}$ nanoionic precipitates. Consequently, the LOD for $\mathrm{Pb}^{2+}$ ion was improved to $\sim 20 \mu \mathrm{M}$ with $[+]-[+/-]_{9}$ Au nanoionic precipitates (green curve in Fig. 4d). The sensitivity of $[+]-[+/-]_{9}$ AuNP nanoionic precipitate is comparable/or even better than other reported NP systems with analyte non-specific ligands (Table S1, ESI †े). More importantly, the plasmon intensity of the redispersed nanoionic precipitates was higher than both of the individual $[+/-]_{9}$ and $[+]$ AuNPs (close to the additive intensity; purple spectrum in Fig. 4d). This confirms the complete redispersal of $[+/-]_{9}-[+]$ nanoionic precipitates in contrast to the partial redispersal of $[+]-[-]$ nanoionic precipitates, which is an important requisite for an ideal detection system. Further, $3 \mathrm{mM}$ of $\mathrm{Cd}^{2+}$ ions as well was able to break the electrostatic interactions in $[+/-]_{9}-[+]$ Au nanoionic precipitates, with a $\sim 50 \%$ revival of plasmon band 
(Figs. 4e and S13, ESI $\dagger$ ). The decrease in the strength of the electrostatic tethers in $[+]-[+/-$ ]$_{9} \mathrm{Au}$ nanoionic precipitates results in a turn-on response for $\mathrm{Pb}^{2+}$ and $\mathrm{Cd}^{2+}$ ions, demonstrating the tunability in the selectivity of toxic ions as per the demand. However, even $3 \mathrm{mM}$ of $\mathrm{Ni}^{2+}, \mathrm{Zn}^{2+}$ and $\mathrm{Ca}^{2+}$ ions failed to break the electrostatic interaction in $[+]$ and $[+/-]_{9}$ Au nanoionic precipitates (Figs. 4e and S13, ESI†).

a)
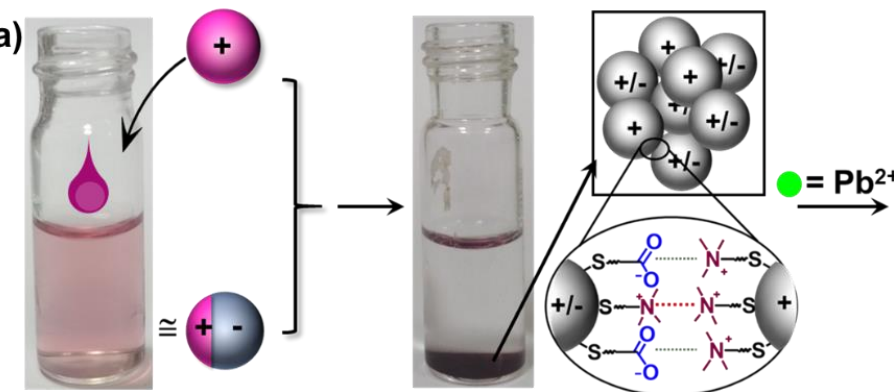

$[+]-[+/-]_{9}$ Au nanoionic ppt.
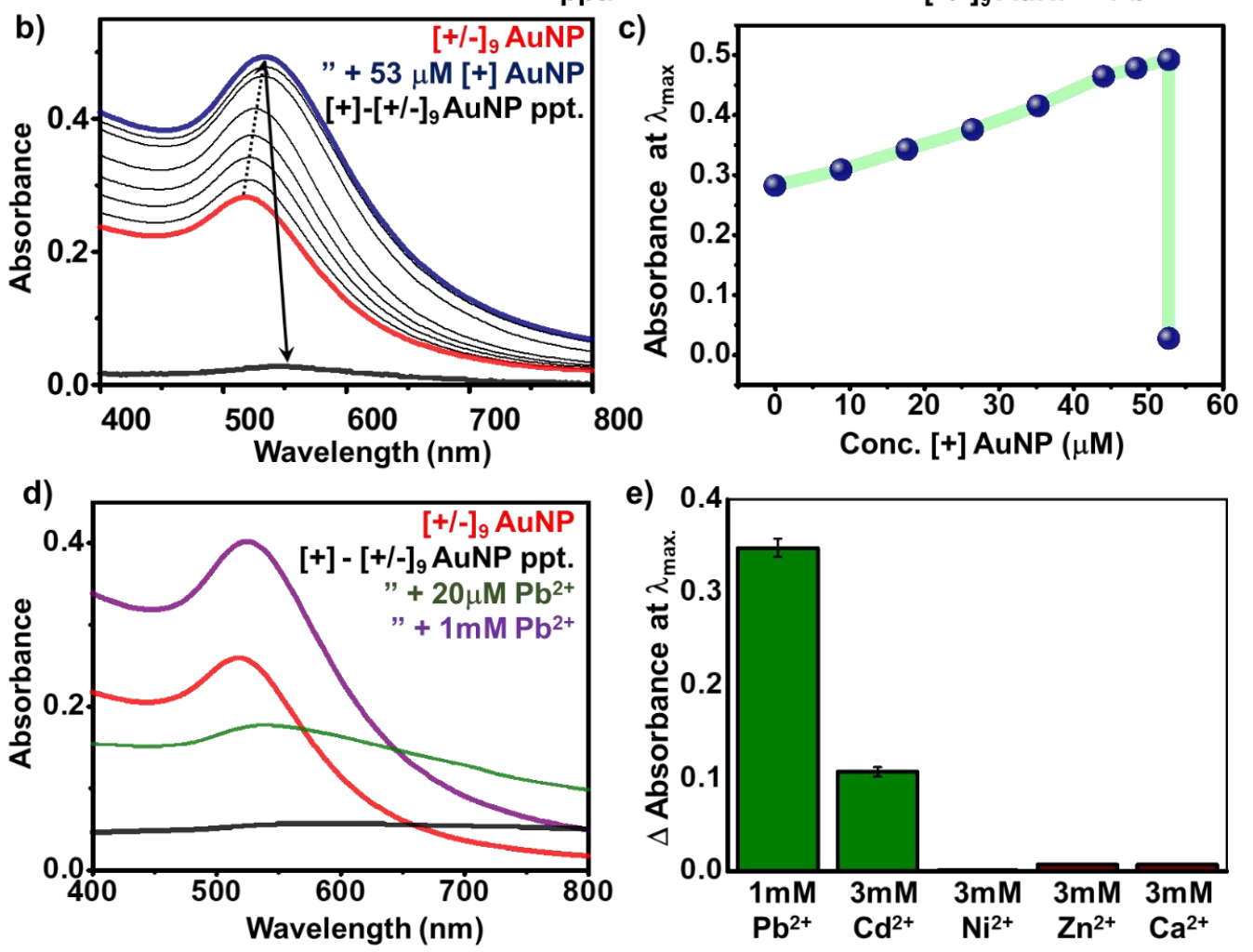

Figure. 4 Versatility and tunability of Au nanoionic precipitates. (a) Scheme for the formation of $[+]$ and $[+/-]_{9}$ Au nanoionic precipitates and amplification in the selective turnon detection of $\mathrm{Pb}^{2+}$ ion. Variation in the (b) absorbance and (c) Absorbance at $\lambda_{\max }$ of $[+/-]_{9}$ AuNP upon addition of [+] AuNP. A sharp decrease in the plasmon band represents precipitation at charge neutrality, confirming the nanoionic behavior. (d) Redispersal of [+] and $[+/-]_{9} \mathrm{Au}$ nanoionic precipitates in the presence of $1 \mathrm{mM} \mathrm{Pb}^{2+}$ ion (with LOD of $20 \mu \mathrm{M}$, green spectrum). The intensity of the revived plasmon band was higher than the individual $[+/-]_{9}$ and $[+]$ AuNPs, confirming the complete redispersal of $[+]-[+/-]_{9}$ Au nanoionic precipitates. (e) The selectivity of $[+]-[+/-]_{9}$ Au nanoionic precipitates in identifying $\mathrm{Pb}^{2+}$ and $\mathrm{Cd}^{2+}$ ions. 
The process of breaking of electrostatic interactions in $[+]-[+/-]_{9}$ Au nanoionic precipitates by $\mathrm{Pb}^{2+}$ ions was further monitored using Atomic Force Microscope (AFM) and Dynamic Light Scattering (DLS) studies. The presence of small and controlled AuNP aggregates (60$180 \mathrm{~nm}$ ) was clearly visible in the AFM images presented in Figs. 5 and S14, ESI $\uparrow$. The 3D AFM height image shows the ripples demarcating the boundaries between the individual NPs constituting the aggregates (Fig. 5c). The AFM results were well complemented by DLS studies, which confirms the breaking of $[+]-[+/-]_{9} \mathrm{Au}$ nanoionic precipitates by $\mathrm{Pb}^{2+}$ to smaller aggregates (Fig. 5d). Moreover, a $\sim 10 \mathrm{~nm}$ bathochromic shift in the $\lambda_{\max }$ of the redispersed sample confirms the formation of controlled aggregates between $[+/-]_{9}$ AuNP and $\mathrm{Pb}^{2+}$ ions (Fig. 4d) ${ }^{23}$ Thus, the stability and dispersion of $[+/-]_{9}$ AuNP $-\mathrm{Pb}^{2+}$ complex is attributed to the perfect balance between attractive and repulsive interactions in the controlled aggregates, as reported previously. ${ }^{23}$

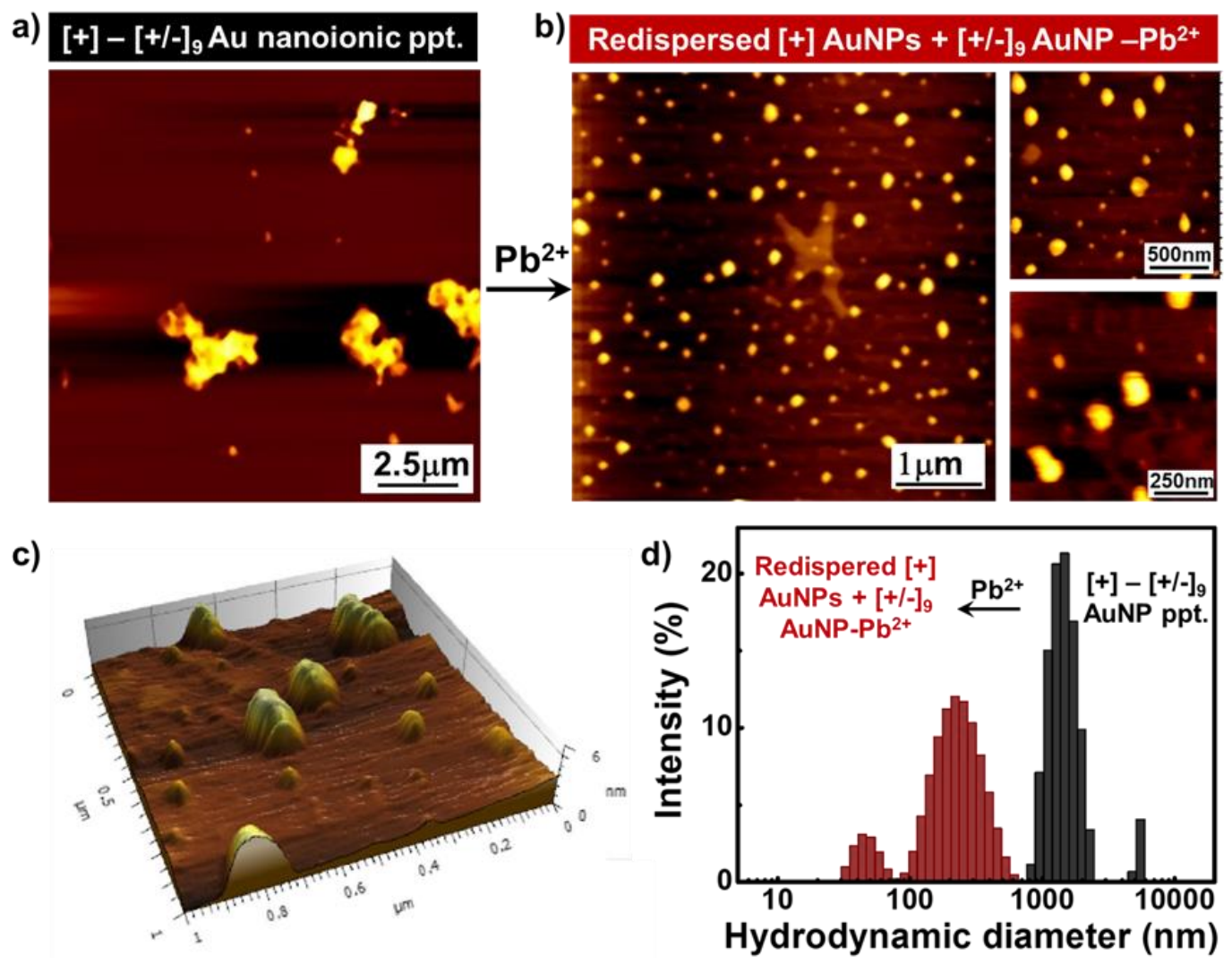

Figure. 5 AFM and DLS proof for the breaking of electrostatic interactions in $[+]-[+/-]_{9}$ Au nanoionic precipitates by $\mathbf{P b}^{2+}$ ions. AFM images of $[+]-[+/-]_{9}$ Au nanoionic precipitates (a) before and (b) after the addition of $1 \mathrm{mM} \mathrm{Pb}^{2+}$ ions. (c) $3 \mathrm{D}$ AFM height image showing the ripples demarcating the boundaries between the individual NPs constituting the aggregates. (d) The DLS data showing the decrease in the hydrodynamic diameter from a few microns to $60-180 \mathrm{~nm}$, upon the addition of $\mathrm{Pb}^{2+}$ ions. 


\section{Conclusions}

This work presents a straightforward, yet effective strategy to transform the commonly observed nonselective turn-off response of [-] AuNP into an attractive selective turn-on response for the identification of heavy metal ions. The difference in the abilities of various $\mathrm{M}^{2+}$ ions to break the interactions between the oppositely charged AuNPs (nanoionic precipitates) was chosen as the means of discrimination, rather than the conventional concept of forming an interaction. Among various $\mathrm{M}^{2+}$ ions tested, $\mathrm{Pb}^{2+}$ was solely able to break the electrostatic interactions in [+] - [-] Au nanoionic precipitates. The displaced [+] AuNPs imparted the characteristic wine-red color to the solution, resulting in a turn-on response. The favorable interaction energy for [-] AuNP - $\mathrm{Pb}^{2+}$ complexation is accountable for the discrimination of $\mathrm{Pb}^{2+}$ from other $\mathrm{M}^{2+}$ ions, including the $\mathrm{Cd}^{2+}$ ions. The fine tuning of electrostatic interactions in the nanoionic precipitates helped in enhancing the $\mathrm{Pb}^{2+}$ ion sensitivity, along with a complete re-dispersal of both the sets of AuNPs. The flexibility and tunability of identification was demonstrated by extending the selectivity towards $\mathrm{Cd}^{2+}$ ion using $[+]-[+/-]_{9}$ Au nanoionic precipitates. Ability to control the electrostatic and bridging interactions was crucial in imparting selectivity to a nanohybrid system composed of constituents that are inherently nonselective. The concept of breaking 'known' strengths of interactions through displacement reaction can help in ascertaining the strengths of 'unknown' interactions, which can find far reaching applications in fundamental as well as applied areas of material chemistry.

\section{Conflicts of interest}

There are no conflicts to declare.

\section{Acknowledgements}

This work was supported by the funding from DST-SERB Grant No. EMR/2015/001561. We thank Dr. Anirban Hazra for useful discussions. A. R. thanks CSIR; G. S., G. D., A. T. R. thanks MHRD and S. R. thank UGC for fellowships.

\section{References}

1. B. A.Grzybowski and W. T. S. Huck, Nat. Nanotechnol., 2016, 11, 585.

2. C. A. Batista, R. G. Larson and N. A. Kotov, Science, 2015, 350, 1242477.

3. K. J. M. Bishop, C. E. Wilmer, S. Soh and B. A. Grzybowski, Small, 2009, 5, 1600.

4. Y. Min, M. Akbulut, K. Kristiansen, Y. Golan and J. Israelachvili, Nat. Mater., 2008, 7, 527. 
5. P. K. Kundu, D. Samanta, R. Leizrowice, B. Margulis, H. Zhao, M. Börner, T. Udayabhaskararao, D. Manna and R. Klajn, Nat. Chem., 2015, 7, 646.

6. J. Kim, M. Han, M. Lien, S. Magonov, Y. Zhu, H. George, T. B. Norris and N. A. Kotov, Sci. Adv., 2018, 4, e1700682.

7. M. A. Boles, M. Engel and D. V. Talapin, Chem. Rev., 2016, 116, 11220.

8. G. A. DeVries, M. Brunnbauer, Y. Hu, A. M. Jackson, B. Long, B. T. Neltner, O. Uzun, B. H. Wunsch and F. Stellacci, Science, 2007, 315, 358.

9. Y. Taniguchi, M. A. B. Sazali, Y. Kobayashi, N. Arai, T. Kawai and T. Nakashima, ACS Nano, 2017, 11, 9312.

10. P. Pramod, S. S. Joseph and K. G. Thomas, J. Am. Chem.Soc., 2007, 129, 6712.

11. S. Roy, A. Rao, G. Devatha and P. P. Pillai, ACS Catal., 2017, 7, 7141.

12. H. Zhao, S. Sen, T. Udayabhaskararao, M. Sawczyk, K. Ku, D. Manna, P. K. Kundu, J. Lee, P. Král and R. Klajn, Nat. Nanotechnol., 2015, 11, 82.

13. G. Devatha, S. Roy, A. Rao, A. Mallick, S. Basu and P. P. Pillai, Chem. Sci., 2017, 8, 3879.

14. J. Chen, S. M. Andler, J. M. Goddard, S. R. Nugen and V. M. Rotello, Chem. Soc. Rev., 2017, 46, 1272-1283.

15. R. Mout, D. F. Moyano, S. Rana, and V. M. Rotello, Chem. Soc. Rev., 2012, 41, 2539.

16. D. A. Giljohann, D. S. Seferos, W. L. Daniel, M. D. Massich, P. C. Patel and C. A. Mirkin, Angew. Chem. Int. Ed., 2010, 49, 3280.

17. A. Verma and F. Stellacci, Small, 2010, 6, 12.

18. A. Le Ouay and F. Stellacci, Nano Today, 2015, 10, 339.

19. P. P. Pillai, B. Kowalczyk, K. Kandere-Grzybowska, M. Borkowska and B. A. Grzybowski, Angew. Chem. Int. Ed., 2016, 55, 8610.

20. Z. V. Feng, I. L. Gunsolus, T. A. Qiu, K. R. Hurley, L. H. Nyberg, H. Frew, K. P. Johnson, A. M. Vartanian, L. M. Jacob, S. E. Lohse, M. D. Torelli, R. J. Hamers, C. J. Murphy and C. L. Haynes, Chem. Sci., 2015, 6, 5186.

21. J. Moratz, A. Samanta, J. Voskuhl, S. K. M. Nalluri and B. J. Ravoo, Chem. Eur. J., 2015, 21, 3271 .

22. Y. Kim, R. C. Johnson and J. T. Hupp, Nano Lett., 2001, 1, 165.

23. A. Rao, S. Roy, M. Unnikrishnan, S. S. Bhosale, G. Devatha and P. P. Pillai, Chem. Mater., 2016, 28, 2348.

24. K. Saha, S. S. Agasti, C. Kim, X. Li and V. M. Rotello, Chem. Rev., 2012, 112, 2739. 
25. D. Jimenez de Aberasturi, J. M. Montenegro, Ruiz de I. Larramendi, T. Rojo, T. A. Klar, R. Alvarez-Puebla, L. M. Liz-Marzán and W. J. Parak, Chem. Mater., 2012, 24, 738.

26. P. Atkins, T. Overton, J. Rourke, M. Weller, F. Armstrong, Shriver \& Atkins' Inorganic Chemistry, Oxford University Press, Great Britain, 2010, Ch-4, pp-119.

27. S. Özkar, In New and Future Developments in Catalysis Batteries, Hydrogen Storage, ed. S. L. Suib, Elsevier, Amsterdam, 1st Edition, 2013, Ch-7, pp-167.

28. S. V. Sokolov, E. Kätelhön, and R. G. Compton, J. Phys. Chem. C 2015, 119, 25093.

29. J. J. Storhoff, R. Elghanian, R. C. Mucic, C. A. Mirkin and R. L. Letsinger, J. Am. Chem. Soc. 1998, 120, 1959.

30. G. Sener, L. Uzun and A. Denizli, ACS Appl. Mater. Interfaces, 2014, 6, 18395.

31. E. A. Baldauff and J. M. Buriak, Chem. Commun., 2004, 2028.

32. K. Yoosaf, B. I. Ipe, C. H. Suresh and K. G. Thomas, J. Phys. Chem. C, 2007, 111, 12839.

33. P. K. Sudeep, S. S. Joseph and K. G. Thomas, J. Am. Chem. Soc. 2005, 127, 6516.

34. G. J. Lumetta, B. M. Rapko, P. A. Garza, B. P. Hay, R. D. Gilbertson, T. J. Weakley and J. E. Hutchison, J. Am. Chem. Soc., 2002, 124, 5644.

35. X. Lin, A. P. Ivanov and J. B. Edel, Chem. Sci., 2017, 8, 3905.

36. M. Rana, M. Balcioglu, N. M. Robertson, M. S. Hizir, S. Yumakc and M. V. Yigit, Chem. Sci., 2017, 8, 1200.

37. Y. Ben-Amram, R. Tel-Vered, M., Riskin, Z. G., Wang and I. Willner, Chem. Sci., 2012, $3,162$.

38. (a) J. Liu and Y. Lu, J. Am. Chem. Soc., 2003, 125, 6642; (b) J. Liu and Y. Lu, J. Am. Chem. Soc., 2004, 126, 12298; (c) J. H. Lee, Z. Wang, J. Liu and Y. Lu, J. Am. Chem. Soc., 2008, 130, 14217.

39. L. Shu-Yi, W. Sung-Hsun and C. Chun-hsien, Angew. Chem. Int. Ed., 2006, 45, 4948.

40. B. Kowalczyk, D. A. Walker, S. Soh and B. A. Grzybowski, Angew. Chem. Int. Ed., 2010, 49, 5737.

41. A. M. Kalsin, B. Kowalczyk, S. K. Smoukov, R. Klajn and B. A. Grzybowski, J. Am. Chem. Soc., 2006, 128, 15046.

42. N. R. Jana and X. Peng, J. Am. Chem. Soc., 2003, 125, 14280.

43. P. P. Pillai, S. Huda, B. Kowalczyk and B. A. Grzybowski, J. Am. Chem. Soc., 2013, 135, 6392.

44. M. J. Eibling, C. M. MacDermaid, Z. Qian, C. J. Lanci, S. J. Park and J. G. Saven, J. Am. Chem. Soc., 2017, 139, 17811. 
45. Y. Wei, K. J. M. Bishop, J. Kim, S. Soh and B. A. Grzybowski, Angew. Chem. Int. Ed., 2009, 48, 9477.

46. K. J. M. Bishop, B. Kowalczyk and B. A. Grzybowski, J. Phys. Chem. B, 2009, 113, 1413.

47. K. J. M. Bishop and B. A. Grzybowski, ChemPhysChem, 2007, 8, 2171.

48. S. L. Carnie, D. Y. C. Chan and J. S. Gunning, Langmuir, 1994, 10, 2993.

49. S. Gobom, Nature, 1963, 197, 283.

50. E. Ferrell, J. M. Ridgion and H. L. Riley, J. Chem. Soc., 1934, 1440.

51. P. P. Pillai, B. Kowalczyk, W. J. Pudlo and B. A. Grzybowski, J. Phys. Chem. C, 2016, 120, 4139. 